\title{
Helicopter Laboratory Model Experiment with Web Access
}

\author{
$\underline{\text { doi:10.3991/ijoe.v5s1.1015 }}$ \\ V.M. Cvjetkovic, M. S. Matijevic, M. D. Grujovic and M. Z. Stefanovic \\ University of Kragujevac, Kragujevac, Serbia
}

\begin{abstract}
This paper describes the experiment for learning and investigation of the helicopter laboratory model static and dynamic characteristics. Helicopter model is not the free flying one, as it has the two degrees of freedom - up / down movement and rotation with respect to vertical axis of the main propeller. The two basic different kinds of experiments with the helicopter model are possible. In the first group are experiments with the model for measurement of the static and dynamic characteristics that show the basic capabilities and overall performance of the model. Second group of experiments are control experiments for movement of the system to the desired position or for performing some continuous oscillatory movement. All experiments can be performed locally from the lab with the experimental setup and remotely. Remote experiments are performed by using the web user interface for controlling the laboratory equipment and the IP camera for observing the movements of the helicopter model.
\end{abstract}

Index Terms-Remote experiment, mechatronics, helicopter, web laboratory.

\section{INTRODUCTION}

Helicopters are among the most interesting and sophisticated means of transport. Their unique maneuvering possibilities make them an attractive and also very instructional "toy" for learning the principles of flight and flight control on the laboratory helicopter model. Laboratory helicopter model used in this experiment was developed primarily for educational purposes of the mechanical engineering students. Achieved benefits with the helicopter model are many and include:

a. active student work during the development and constructional phase

b. experiments for measurement of the static and dynamic characteristics of the helicopter laboratory model

c. "free" control and management of the helicopter model using manual controls, i.e. playing and learning control

d. Design and implementation of the controllers that act as safety limiters in the case of inadequate and improper manual control of the inexperienced or user with bad intentions

e. Experimenting with automated flights - programmed flights that are completely controlled by the software.

In addition, all mentioned items b) - e) can be performed locally and remotely, using the RemoteLab [1] infrastructure that was developed previously. However, remote experiments with helicopter model are specific and different comparing to other previous web experiments, as they last much longer, and are much more interactive and thus substantially require video control for the user manual control of the helicopter model when used remotely, and for observing of the helicopter model behavior.

Construction of the laboratory helicopter model required knowledge from various disciplines of the technical sciences, such as mechanics, electronics and programming, which are all together uniquely known as the mechatronics.

\section{HELICOPTER MODEl CONSTRUCTION}

Construction of the helicopter model was performed in phases, directly relating to the well known procedure consisting of analysis, design, implementation, testing and system usage - exploitation.

In the phase of analysis, the most important considerations included the choice of the helicopter model. First, the detailed study was performed for the theory of the helicopter flight principles [2]. There are two basic kinds of the RC (Radio Controlled) helicopter models [3], regarding the technical solution for the propeller, and can be with fixed or changing pitch, which is the attack angle of the main propeller. Also, there are two different possible technical constructions for the compensation of the not desired helicopter rotation in the opposite direction of the main propeller rotation due to the angular momentum preservation. One technical solution is to produce the opposite momentum by the tail propeller as in the vast majority of helicopters, and the other is with the two main propellers on the same shaft rotating in the opposite directions, thus canceling the momentums. The other solution with the two propellers on the same axis, rotating in the opposite directions is known as coaxial rotor also called the Kamov design [4].

Some other important constructional facts relate to possible movements of the helicopter model, i.e. the system degrees of freedom. As this was an undergraduate student project, system with the two degrees of freedom was chosen. It can still serve for demonstration of the basic helicopter flight characteristics with very simple construction of both propellers in one piece and made of wood. Such design and make is quite enough for the two degrees of freedom of the helicopter model. The other important characteristic is the compensation of the main

propeller torque. As it was technically much simpler to implement classical design with just one main propeller, the most frequent solution was chosen with the one main horizontal propeller and one vertical in the tail for the main horizontal propeller torque compensation. Fig. 1 
shows the helicopter model degrees of freedom and the basic constructional details.

As can be seen from Fig. 1, both degrees of freedom are measured with rotation angles $\alpha$ and $\beta$. The two lever mechanism provides the vertical movement measured by the angle $\alpha$, while the yaw rotation of the helicopter is measured by the angle $\beta$. On the opposite side of the helicopter are the two weights for balancing the helicopter mass. The purpose of balancing is to enable the vertical movement, as the main propeller thrust is not enough to move the helicopter upwards without the balancing weights. Balancing weights on the other side increase the overall mass of the system, making it more inert to changes of the main propeller thrust, i.e. to changes of the height - vertical movement. Separate electric motors drive the main and the tail propeller. The two angles $\alpha$ and $\beta$ are measured by the rotational potentiometers that convert the angular position into proportional dc voltage. Fig. 2 presents the whole helicopter model mounted on the fixed stand. Maximal measured force of the thrust produced by the main propeller is around $2 \mathrm{~N}$.

\section{THE BASIC HELICOPTER CONTROLS}

Real world helicopters have four basic controls. These are called the cyclic, the collective, the anti-torque, and the throttle. Cyclic control of the helicopter is the most complex one that is responsible for the unique maneuvering capabilities of the helicopter. Cyclic controls change the tilt or the pitch of the propeller in cyclic manner for each turn of the propeller. Cyclic commands are translated by the swash plate device to motion of main propeller blades. Fig 3 shows the swash plate of the RC helicopter.

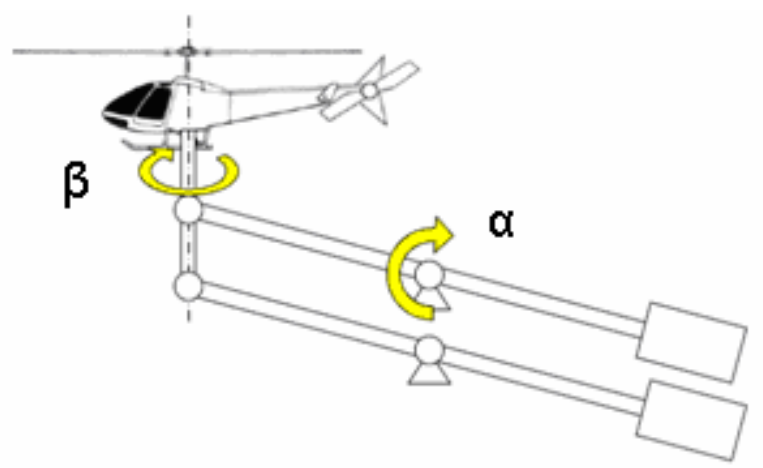

Figure 1. Degrees of freedom of the helicopter model

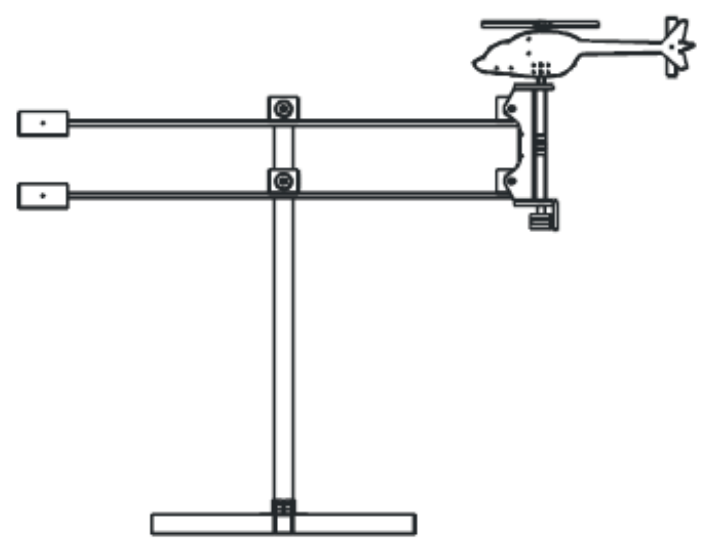

Figure 2. Helicopter model on the fixed stand

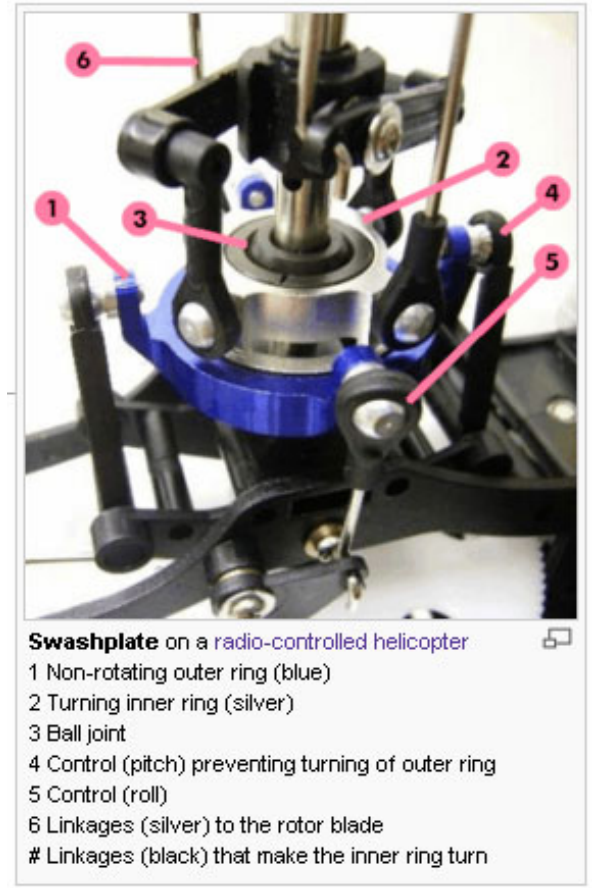

Figure 3. Swash plates of the RC helicopter

The collective control changes the pitch angle of all the main rotor blades collectively (i.e. all at the same time) and independently of their position. Therefore, if a collective input is made, all the blades change equally, and the result is the helicopter increasing or decreasing in altitude.

Anti torque controls the thrust produced by the tail propeller to compensate the torque produced by the rotation of the main propeller.

Throttle controls the lift force - thrust produced by the main propeller that keeps the helicopter in the air. It changes the rotational speed of the main propeller. The thrust can also be changed by the collective pitch.

Helicopter laboratory model with 2 degrees of freedom has only two of the mentioned controls, i.e. the throttle and anti torque. Throttle controls the thrust by changing the rotation speed of the main propeller, while the anti torque control changes the rotation speed of the tail propeller.

\section{MEASUREMENTS ON THE HELICOPTER LABORATORY MODEL}

The main purpose of the helicopter laboratory model besides learning the constructional details and used technical solutions, are various kinds of measurements that can be performed on it. All measurements can be divided into the following groups:

a. Determining the static characteristics

b. Determining the dynamic characteristics

c. Measurements with various kinds of controllers

d. Manual control of the laboratory model for testing and flight practicing

e. Programmed flight of the helicopter, when the helicopter model performs series of movements defined and initiated by the computer program.

Each of the previous group of measurements can be further elaborated. 


\section{A. Measurements for determining of the static characteristics}

Static characteristic expresses static functional dependence between the two or more values that are frequently of different physical quantities, but not necessarily. Static means that the measurements are made in physical equilibrium, or with very slow changes that approach equilibrium state well. Usually, it is desirable for the static characteristic of any kind to be linear. With given helicopter model, a few static characteristics of practical interest can be defined.

a. Functional dependence of the rising angle to voltage applied on the electric motor for the main propeller.

b. Functional dependence of the rotation speed to voltage applied on the electric motor for the tail propeller.

c. Functional dependence of the voltage applied to electric motor for the main propeller to voltage applied to electric motor of the tail propeller, for which there is no rotation of the helicopter around vertical axis, i.e. for compensated torque

Necessary measurements can be easily performed, by measuring angles and voltages and writing down the measured values to data file for calculation of the characteristics using some mathematical method.

Determining the previous static characteristics is of practical use for the design of controllers, and programmed flight.

\section{B. Measurements for determining of the dynamic characteristics}

Quite analogous to static characteristics, similar dynamic characteristics can be defined, but this time not in conditions of physical equilibrium. Some standard input functions - step function, rectangular pulse, harmonic function, triangular, saw tooth or some other can be applied for the measurement of the dynamic characteristics response of the system. Dynamic behavior of the system is governed by the system order - whether it is of the $1^{\text {st }}, 2^{\text {nd }}$ or of the higher system order. Dynamic behavior of the helicopter model can be considered independently for the vertical and horizontal movement - rotation and joined for both movements.

1. Dynamic characteristics for vertical movement

2. Dynamic characteristics for horizontal movement

3. Dynamic compensation of rotation with tail propeller

Determining of dynamic characteristics for the helicopter model can be directly used for the design of various controllers.

\section{Measurements with various kinds of controllers}

Helicopter as sophisticated flying device has manual controls that enable intentional usage of the helicopter by the human operator - pilot. But as for any other technical system that can be controlled, many various controllers can be designed. Those controllers can be for the support of the main operator, such as servo devices, or for control of some critical operator activities and avoidance of inadequate human operator actions that could lead to the instability of the system. Such controllers should serve primarily as an aid to more efficient human control of the system. For the simple helicopter model under consideration, the two types of controllers can be designed.

1. Controller for helicopter height stabilization that can neutralize air disturbances leading to height variations

2. Controller for horizontal rotation stabilization that can neutralize eventual rotation in the horizontal plane caused by the changes of rotation speed of the main propeller

Proposed controllers types can be designed and implemented using various kinds of controllers.

\section{Manual control of the helicopter model}

As already mentioned, helicopters are intended for manual control by the human operators - pilots. Therefore, it is good to have adequate manual controls for the helicopter model that can provide the student with the ability to manually control the model, in order to get the feeling of control, and to learn the correct manual actions. In that case human operator acts as a controller, and closes the feedback loop. Various combinations of helicopter movements can be tried.

\section{E. Programmed flight}

In contrast with the manual control of the helicopter model, is the fully operated automatic control of the model "flight". There is some similarity with previously mentioned controllers, but the difference is that the control is dynamic, performing desired movements of the helicopter model, instead of trying just to stabilize it when the disturbances change the desired controlled values. One way to achieve that is to design the controller that will have as its input the time dependant - changing reference value. The changing value with time of the controlled reference is programmed, i.e. it changes with time according to some pre determined function of time. For the two degrees of freedom helicopter model, that would include the time functions of angles $\alpha$ and $\beta$ to define the desired movements of the helicopter model. Programmed flight can be thought of as the next higher step comparing to an ordinary control of the constant values.

Each of the mentioned five groups of measurement experiments can be saved to file for later analysis.

\section{EXPERIMENTAL SYSTEM ARCHITECTURE}

Experimental system built around the helicopter model has the main function to enable the local and remote users to work with the model, i.e. to make experiments and measurements mentioned in the previous chapter. The basic system experimental architecture closely resembles the architecture in RemoteLab system developed earlier [1]. Experimental setup - architecture of the system with laboratory helicopter model is shown in Fig. 4, as UML node - deployment diagram of the system.

Helicopter laboratory model is controlled by the acquisition PC using the data acquisition system (DAS). DAS [5] provides 2 input analog channels for voltage measurement on angle to voltage potentiometer converters, and the two output channels for controlling the two electric motors. Not shown in Fig. 4, is the electronic amplification system for controlling the electric motors with the low current analog voltage outputs. Data acquisition PC runs the LabVIEW 8.5 [6] software for controlling the 


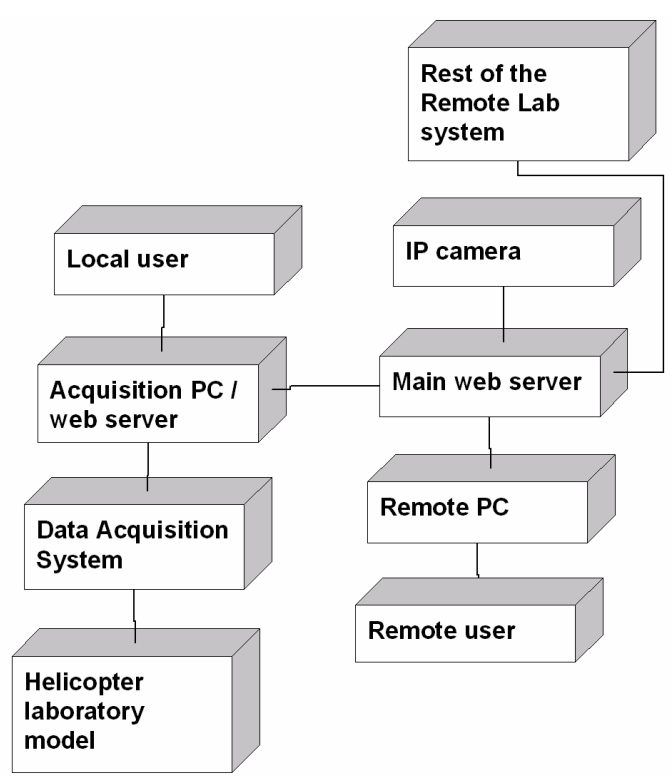

Figure 4. UML node diagram of the system

DAS. All applications for this experiment are created by LabVIEW. LabVIEW software provides the adequate graphical user interface with controls and indicators needed in the experiment. Local user performs experiments using that interface from the acquisition PC. For remote users, LabVIEW provides the web server running on the acquisition PC. Remote user can then access the same interface over network from web browser on remote PC. In order to connect to experiment, remote PC must have the LabVIEW run time engine installed with version number matching the version number of LabVIEW used for the experiment. IP camera is used for viewing the experiment setup from the remote user browser. The main web server contains web pages for remote access to the whole system. Specific web pages provide support for the helicopter model experiments.

Web pages on the main web server also combine the remote user interface of the experiment with the live picture from the IP camera that shows what is actually happening with experimental setup - helicopter model. The node named "Rest of the Remote Lab system" comprises all other previously developed and implemented experiments with the similar structure.

\section{MEASUREMENTS PERFORMED}

Some of the measurements described in chapter IV are presented here as an example of the system functioning. All the experiment examples shown here are with the remote - web access to experiments. Web pages that present the experiments always consist of two parts. On the left is the user interface from the corresponding LabVIEW Virtual Instrument (VI), and on the right is the live image from the IP camera showing the actual movements of the experimental setup with the helicopter model according to signals sent from the user interface with the chosen experiment.

\section{A. Static characteristic measurement}

Fig. 5 shows the web page for the measurement of the helicopter static characteristic that connects the input voltage for the main propeller with the angle that determines the height of the model, the angle $\alpha$.

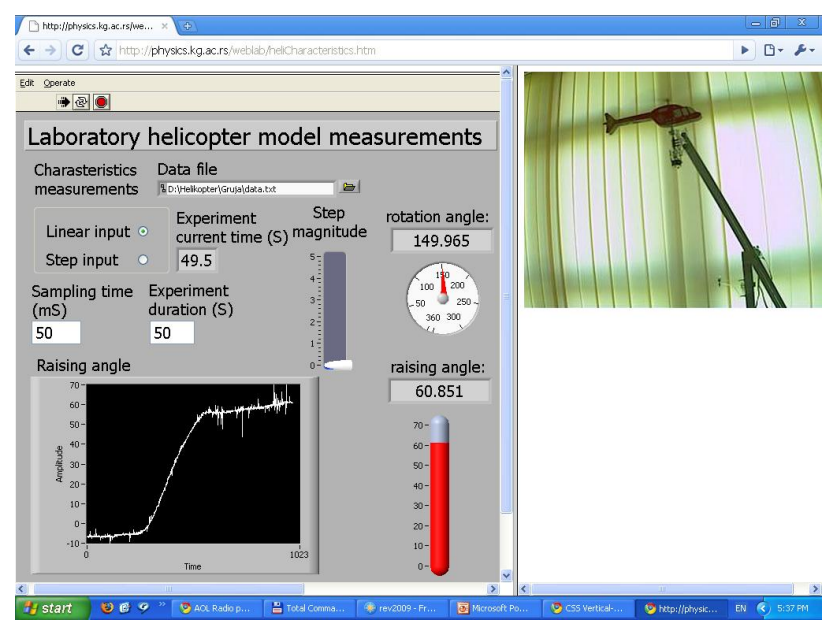

Figure 5. Remote measurement of static characteristic

Type of the characteristic measured can be determined with "Linear input" / "Step input" selection. With the "Linear input" selected, input voltage changes from the min, $0 \mathrm{~V}$ to $\max 5 \mathrm{~V}$ during the time specified in "Experiment duration (S) input field, with sampling time in "Sampling time (mS)" input field. Experiment duration should be chosen to be long enough, to provide the quasi equilibrium state during the measurement. Measured values are written to data file for later calculations. Left horizontal part on the diagram for "Raising angle" is caused by the too low voltage below the threshold for moving the model upwards. Horizontal part on the right starts with the voltage that brings the model into max possible vertical position - saturation.

\section{A. Dynamic characteristic measurement}

Measurement of the dynamic characteristic can be performed from the same web page, just by selecting the

"Step input" option instead of "Linear input" for the static measurement. Step magnitude can be adjusted using the sliding control with the same name. Adjustment of the "Step magnitude" prevents the possible saturation with input driving the system above max. possible position. Fig. 6 shows the same web page as on Fig. 5, but this time with "Step input" selected, which gives the different measured diagram.

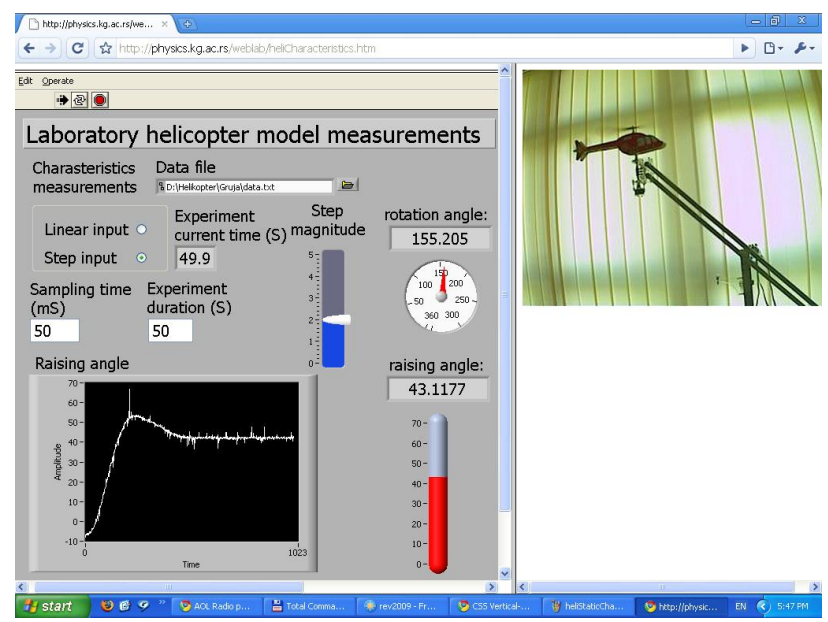

Figure 6. Dynamic characteristics measurement 


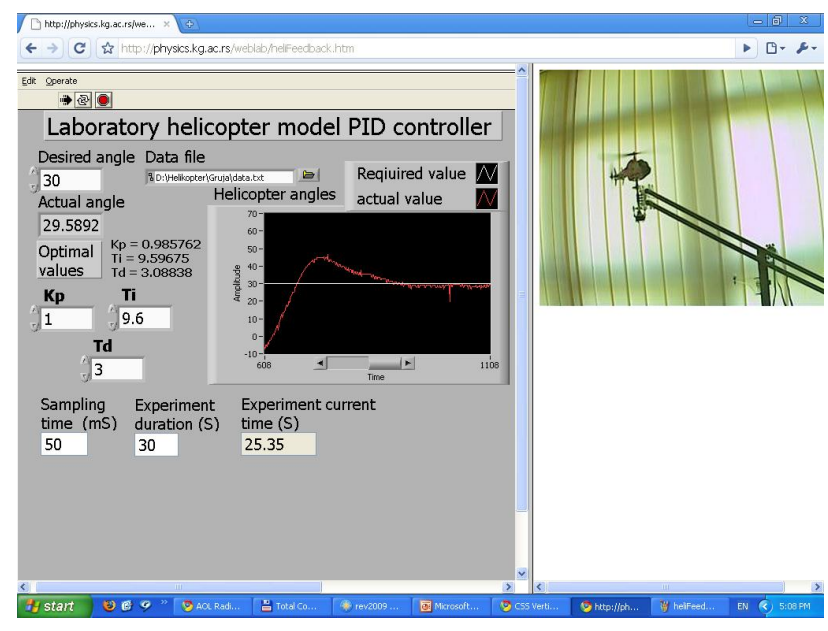

Figure 7. PID experiment

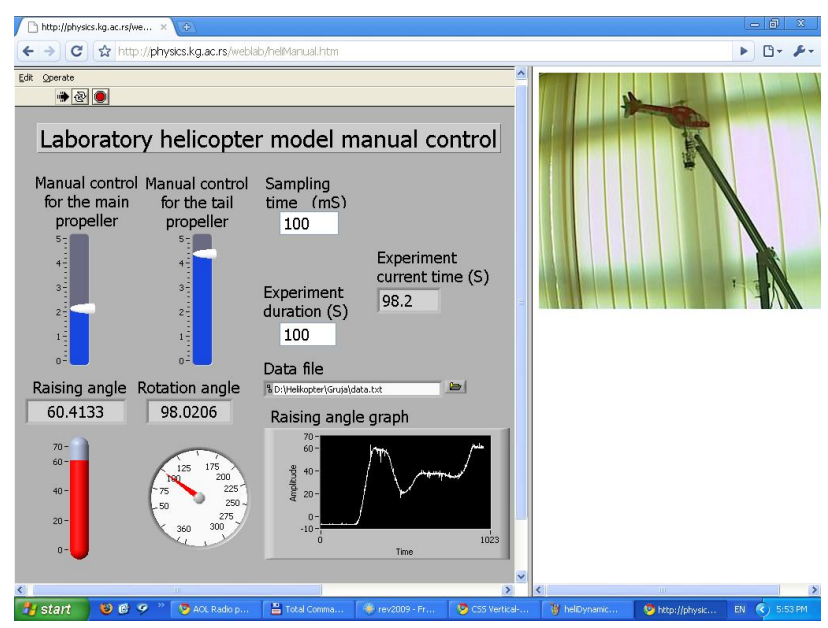

Figure 8. Manual control of the model

\section{B. Experiment with PID controller}

PID controller is used for height regulation of the helicopter model. Fig. 7 shows the web page for PID controller. The desired angle is given in the input field with the same name. Experiment starts from the lowest position of the model, rising to the desired stabilized position.

\section{Manual control}

Manual control is the experimental option for users that would like to try the direct control of the helicopter model, when the user itself acts as controller. Feedback, or the control loop in that case is closed with IP camera that shows the current position of the model, so the user is able to see the movements of the model as he drags the sliders for manual control of the main propeller and the tail propeller. IP [7] camera transfers 10 picture frames per second, which is enough for almost continuous impression of the model movements. Number of picture frames from the IP camera is controlled by the JavaScript software that uses AJAX technique for changing the picture from the IP camera only, without resending the whole web page.

Number of picture frames per second from the IP camera can be easily increased to any desired value, up to 30 images per second in resolution of $640 \times 480$. Fig. 8 shows the web page for manual control of the model.

\section{VII.CONCLUSION}

Laboratory helicopter model with two degrees of freedom was developed for educational purposes of mechanical engineering students. Many different measurements grouped in the five types of experiments can be performed with laboratory helicopter model both locally and remotely. Remote access to web experiments give the opportunity to a much larger number of students to access, use and learn with once developed and tested experiment setup, comparing to traditional local experiments. Experiments are designed so that the lack of the presence in the lab with experimental setup does not make any important obstacle for making proposed experiments remotely. Experiment with the laboratory helicopter model was included as the new module to already existing system with web experiments.

\section{ACKNOWLEDGMENT}

Authors are grateful to M.S. Miroslav Ravlić, Ph.D. student of the Faculty of Mechanical engineering, for help in construction of the helicopter laboratory model.

\section{REFERENCES}

[1] V. M. Cvjetkovic, Y. S. Sulema, Remote Laboratory for Supporting e-Studies in Electronics, iJoe,Volume 2, No. 1, 2006

[2] Seddon, J., Basic Helicopter Aerodynamics, BSP Professional Books, Oxford, 1990

[3] http://www.electric-rc-helicopter.com/

[4] Rees, Elfan ap, World Military Helicopters, Jane's, London, 1986

[5] http://www.tau.ac.il/ electro/pdf files/computer/ni 6008 ADC $\underline{\text { manual.pdf }}$

[6] http://www.ni.com/labview/

[7] http://www.axis.com/products/cam_206/

\section{AUTHORS}

V. M. Cvjetkovic is with the Faculty of Science, Department of Physics, University of Kragujevac, Serbia, vladimir@kg.ac.rs

M. S. Matijevic, is with the Faculty of Mechanical engineering, University of Kragujevac, Serbia, matijevic@kg.ac.rs

M. D. Grujovic is with the Faculty of Mechanical engineering, University of Kragujevac, Serbia, milan.grujovic@gmail.com

M Z. Stefanovic is with the Faculty of Mechanical engineering, University of Kragujevac, Serbia, miladin@kg.ac.rs

This work was supported in part by the TEMPUS JEP 41029-2006 Interdisciplinary Engineering Education Establishment.

This article is a modified version of a presentation at the REV2009 conference, held at University of Bridgeport, Bridgeport, CT, USA in June 2009. Submitted, July, 10, 2009. Published as resubmitted by the authors on July, 10, 2009. 